\title{
Modeling and simulation of bone mineral density in Japanese osteoporosis patients treated with zoledronic acid using tartrate-resistant acid phosphatase $5 \mathrm{~b}$, a bone resorption marker
}

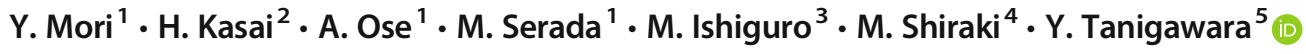

Received: 12 October 2017 / Accepted: 2 January 2018 / Published online: 8 February 2018

(C) The Author(s) 2018. This article is an open access publication

\begin{abstract}
Summary Annual intravenous administration of zoledronic acid is used in the treatment of osteoporosis. A mathematical model was developed to predict bone mineral density up to 2 years after two annual doses of zoledronic acid from the early values of a bone resorption marker in osteoporosis patients.

Introduction The measurement of bone mineral density (BMD) has been used as a surrogate marker instead of the observation of incident fractures to detect the efficacy of treatment. However, this method requires a long time to obtain significant changes. On the other hand, bone resorption markers respond to bone resorption inhibitors within a few weeks. Therefore, the aim of this study was to develop a mathematical model predicting long-term BMD after two annual doses of zoledronic acid (ZOL) using the early response of a bone resorption marker in osteoporosis patients.

Methods The model was constructed using 3410 tartrate-resistant acid phosphatase $5 \mathrm{~b}$ (TRACP-5b) serum concentrations and 1146 lumbar spine (L2-L4) BMD values from 306 patients with primary osteoporosis. A mathematical model was developed to describe the time-dependent profiles of TRACP-5b and BMD.

Results The percentage changes from baseline of the BMD (\%BMD) at up to 2 years were predicted from patients' baseline BMD and baseline and 12-week TRACP-5b values by the model obtained. The simulated $90 \%$ prediction interval almost covered the observed \%BMD distribution at each time point, and the predictions were comparable to the observed \%BMD. Conclusions This is the first model to predict BMD for up to 2 years following two annual doses of ZOL using patients' background characteristics and the early response of TRACP-5b. This model allows us to inform patients at the initial stage of ZOL treatment of their predicted response to treatment.
\end{abstract}

Keywords Bone mineral density · Bone resorption marker · Modeling and simulation · Osteoporosis · Tartrate-resistant acid phosphatase $5 \mathrm{~b}$ (TRACP-5b) - Zoledronic acid

Y. Mori and H. Kasai contributed equally to this work.

Electronic supplementary material The online version of this article (https://doi.org/10.1007/s00198-018-4376-1) contains supplementary material, which is available to authorized users.

Y. Tanigawara

tanigawara-yusuke@umin.ac.jp

1 Development Planning, Clinical Development Center, Asahi Kasei Pharma Corporation, 1-105 Kanda Jinbocho, Chiyoda-ku, Tokyo 101-8101, Japan

2 Certara G.K, 4-2-12, Toranomon, Minato-ku, Tokyo 105-0001, Japan

3 Laboratory for Safety Assessment and ADME, Pharmaceuticals Research Center, Asahi Kasei Pharma Corporation, 632-1 Mifuku, Izunokuni, Shizuoka 410-2321, Japan
4 Department of Internal Medicine, Research Institute and Practice for Involutional Diseases, 1610-1 Meisei, Misato, Azumino, Nagano 399-8101, Japan

5 Department of Clinical Pharmacokinetics and Pharmacodynamics, Keio University School of Medicine, 35 Shinanomachi, Shinjuku-ku, Tokyo 160-8582, Japan 


\section{Introduction}

Osteoporosis, characterized by decreased bone strength [1], is a serious health problem in an aging society, because of its associated high fracture susceptibility. Bone mineral density (BMD) accounts for $70 \%$ of bone strength [1] and is thought to be the standard measure for the diagnosis of osteoporosis and the assessment of fracture risk [2]. BMD is used in the diagnostic criteria for osteoporosis defined by the World Health Organization (WHO); a BMD that lies -2.5 standard deviations or less below the average value for young healthy adults (T-score $<-2.5 \mathrm{SD}$ ) is considered to indicate osteoporosis [3]. Furthermore, it is known that a T-score $>-2.5$ is important for reducing the future fracture risk and would be the goal of osteoporosis treatment $[4,5]$. Therefore, BMD measurement has been considered a good surrogate marker for the occurrence of new osteoporotic fractures. However, the detection of changes in BMD after treatment to the level of the least significant increase requires a long time [6]. On the other hand, changes in bone turnover markers are observed within a few weeks after the start of treatment with bisphosphonates, and the early changes in bone markers predict the long-term response of BMD [7-9]. Diez-Perez et al. proposed that the amino-terminal extension peptide of procollagen type 1 (PINP) and C-telopeptide of type I collagen (CTx) after 3 months of therapy could be used to screen for the treatment effect of oral bisphosphonate therapy [8].

Since a high susceptibility to bone fractures is caused by increased bone resorption, bone resorption inhibitors, especially bisphosphonates, have been used as first-line drugs for preventing osteoporotic fractures. Annual intravenous administration of zoledronic acid (ZOL), a third-generation nitrogen-containing bisphosphonate, has been approved in Japan for the treatment of osteoporosis. In a 2-year, randomized, placebo-controlled study of 665 Japanese patients with primary osteoporosis (ZONE study), the 2-year cumulative incidence of new morphometric vertebral fractures was $3.3 \%$ in the ZOL group versus $9.7 \%$ in the placebo group [10]. In addition, the ZONE study showed that ZOL increased lumbar spine BMD [10].

Over the past decade, many efforts have been made to develop mathematical models to describe the timedependent lumbar spine BMD response to antiosteoporosis agents [11-16]. Two mathematical models to predict the profiles of percentage change from baseline BMD (\%BMD) after denosumab treatment have been developed. One is a bone homeostasis model that integrates denosumab pharmacokinetics with binding to receptor activator of nuclear factor $-\mathrm{KB}$ ligand and ibandronate inhibition of osteoclast precursor differentiation to active osteoclasts and, furthermore, incorporates the activity of bone resorption markers such as CTx and N-telopeptide of type I collagen (NTx) [14, 16]. Another model is a multiscale systems pharmacology model that incorporates calcium and bone homeostasis and osteoclastic activity (measured by $\mathrm{CTx}$ ) and osteoblastic activity (measured by bone-specific alkaline phosphatase) [13, 15]. Other than denosumab, Hasegawa et al. constructed an exposure-response model to describe the BMD response to ONO-5334, a selective inhibitor of cathepsin K [12]. In addition, Nakai et al. developed a population pharmacodynamic model to relate urinary CTx to the BMD response after treatment with ibandronate, adequately predicting \%BMD at 3 years after treatment [11]. These reports have shown the successful training of mathematical models to describe the BMD response at 1 to 4 years after drug treatment. However, these efforts did not focus on the use of bone turnover markers at the initial stage of drug treatment to predict individual BMD responses years later.

Taken together, the aim of the present study was to develop a mathematical model that would adequately predict BMD for up to 2 years following two annual doses of ZOL using the early values of a bone resorption marker.

\section{Methods}

This study was conducted in compliance with the World Medical Association Declaration of Helsinki-Ethical Principles for Medical Research Involving Human Subjects and Good Clinical Practice. The protocol was approved by the institutional review board at each study site. Written, informed consent was obtained from all subjects before enrollment in the study.

\section{Clinical study data}

All data used in this analysis were obtained from a previously reported study [10]. In summary, this was a multicenter, randomized, placebo-controlled, double-blind, parallel-group, comparative, 2-year study conducted in Japan. All patients with primary osteoporosis were randomly allocated to either ZOL $5 \mathrm{mg}$ or the placebo group in a 1:1 ratio. Randomized patients were administered the study drug once yearly by intravenous infusion (over $15 \mathrm{~min}$ ). Data from both groups were used in this analysis. Although 665 patients ( 333 for ZOL; 332 for placebo) were randomized in the study, data from 306 patients (145 for ZOL; 161 for placebo) who had values for at least one serum bone resorption marker value and BMD were analyzed in this study.

The following bone resorption markers were collected under overnight-fasted conditions and measured at baseline and at $1,2,4$, and 12 weeks and $6,12,18$, and 24 months after the first infusion and at 1,2, and 4 weeks after the second infusion. Serum tartrate-resistant acid phosphatase 5b (TRACP- 
5b) was measured with an Osteolinks ${ }^{\mathrm{TM}}$ TRACP-5b kit (DS Pharma Biomedical Co., Ltd. Osaka, Japan), which was a fragment absorbed immunocapture enzymatic assay. This method employs two monoclonal antibodies (anti-active TRACP-5b antibody and anti-inactive TRACP-5b antibody) and enables highly specific measurement of TRACP-5b without cross-reaction with TRACP-5a derived from macrophages with interassay coefficients of variation of $1.8-7.5 \%$ (unpublished data). CTx was determined using an Elecsys ${ }^{\circledR} \beta$ CrossLaps/serum assay (Roche Diagnostics K.K., Tokyo, Japan) with interassay coefficients of variation of 1.8-6.0\% [10]. Urinary NTx (u-NTx) was measured using Osteomark ${ }^{\circledR}$ (Alere Medical Co., Ltd. Tokyo, Japan) with interassay coefficients of variation of 4.7-10.8\% (unpublished data). All samples were measured by LSI Medience Corporation (Tokyo, Japan). Lumbar spine (L2-L4) BMD was measured using dual-energy X-ray absorptiometry (DXA) at baseline and at 6,12 , and 24 months, as previously described [10]. All study sites used the same DXA brand (Hologic Co., Bedford, MA).

\section{Base model for bone resorption markers}

Bone resorption maker models were developed using TRACP-5b, CTx, or u-NTx, respectively, as follows.

In the first step, the drug effects on the profiles of bone resorption markers were directly modeled with the same model as that used by Nakai et al. [11] (Fig. 1), because no drug concentration data were obtained in this study. In this mathematical model, ZOL dosage was linked to the hypothetical "Effect Site" drug amounts (A), which, in turn, affected bone resorption markers. It was postulated that the bone resorption markers were synthesized in a zero-order manner with a constant Kin and eliminated with the first-order rate constant Kout. The drugs in the "Effect Site" were assumed to inhibit the synthesis of bone resorption markers. The mathematical equations for the model are shown below.

$$
\begin{aligned}
& \frac{d A}{d t}=-K D \times A \\
& E F F=\frac{(K D \times A)^{\gamma}}{E K D_{50}^{\gamma}+(K D \times A)^{\gamma}} \\
& \frac{d \text { Marker }}{d t}=\text { Kin } \times(1-E F F)-\text { Kout } \times \text { Marker }
\end{aligned}
$$

$\mathrm{KD}$ is the first-order equilibrium rate constant, and $\mathrm{EKD}_{50}$ corresponds to the value of $\mathrm{KD} \times \mathrm{A}$ that leads to $50 \%$ inhibition of Kin. $\gamma$ is the Hill's coefficient.

Next, the effect of daily oral supplements (calcium $610 \mathrm{mg}$, vitamin D $400 \mathrm{IU}$, and magnesium $30 \mathrm{mg}$ ) and the natural disease progression effect were added to the above calculated marker levels. These two effects could not be independently estimated because all patients received the daily oral

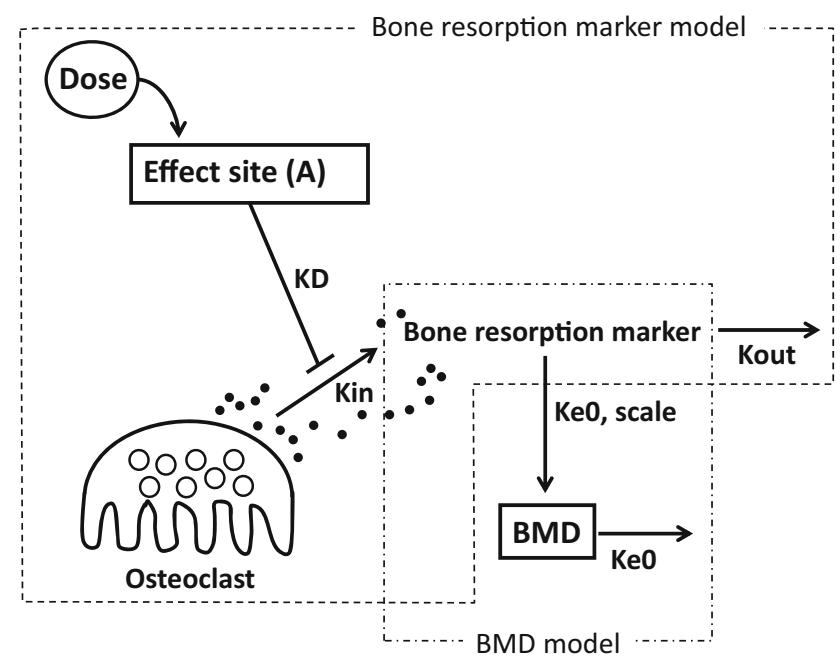

Fig. 1 Schematic description of the mathematical model of ZOL to describe the time-dependent profiles of bone resorption markers and BMD. Effect site hypothetical site where ZOL is stored until the onset of the inhibitory effect, $K D$ first-order equilibrium rate constant, Kin bone resorption marker production rate constant, Kout bone resorption marker elimination rate constant, $\mathrm{KeO}$ effect-compartment equilibrium rate constant, Scale ratio of change in bone resorption marker values to change in $\mathrm{BMD}, B M D$ bone mineral density

supplements; therefore, these effects were simultaneously modeled with the equation shown below.

$\operatorname{Marker}(t)=$ Marker $\times\left(1+\right.$ Slope $\left.\times t+\frac{\text { Emax } \times t}{T_{50}+t}\right)$

where Marker( $\mathrm{t}$ ) is the bone resorption marker at time $\mathrm{t}$ (day), whereas Marker is calculated as above. Slope was a linear time effect, and the last term of the equation showed a timesaturating effect on disease progression, in which Emax was the relative maximal effect, and $\mathrm{T}_{50}$ was the time at which the effect reached half of the maximal effect.

\section{Base model for BMD}

BMD models were developed using TRACP-5b, CTx, or uNTx, respectively.

The mathematical model used in the BMD model is also depicted in Fig. 1. In this model, the time profiles for BMD were assumed to be linearly affected by the bone resorption marker values with a first-order equilibrium constant $\mathrm{Ke} 0$, and they decreased with the same constant. The scale was used to adjust the change in bone resorption marker values to the change in BMD. The mathematical equation for this model is as follows:

$\frac{d B M D}{d t}=K e 0 \times[$ Scale $\times($ Marker-Marker 0$)-(B M D-B M D 0)]$

where Marker0 and BMD0 are the baseline values for the bone resorption markers and BMD, respectively. The bone 
resorption marker for this model development was selected from among TRACP-5b, CTx, and u-NTx based on statistical significance testing.

\section{Statistical models}

The inter-individual variabilities for the model parameters both for the bone resorption markers and the BMD models were assumed to be normally or log-normally distributed with mean 0 and variances $\omega^{2}$, in which parameters with positive values were modeled by a log-normal distribution, and those with positive and negative values were modeled with a normal distribution. Correlations between variabilities of two random effect parameters were also assumed. Intra-individual variability for the bone resorption markers was assumed to follow a relative error model with a standard deviation $\sigma$. Intraindividual variability for the BMD was modeled with absolute error.

Covariate models for each model were also tested using the following patient characteristics: sex, age, body weight, prior usage of some bisphosphonates, and baseline bone resorption markers and BMD. Continuous covariates were modeled by the power model with standardization to their median values, and the power coefficients were estimated. Categorical covariates such as sex and prior bisphosphonate usage were modeled in a relative effect manner.
The likelihood ratio test using a forward inclusion or a backward elimination process was used to compare nested models and select the final model. The full model was developed by incorporating the statistically significant candidates after the forward inclusion process. Each candidate was then tested in turn by removing them one by one to confirm their statistical significance. A minus twice log-likelihood difference was used to judge the statistical significance of the candidate covariates. The $p$ values for the forward inclusion process and the backward elimination process were $<0.05$ and $<$ 0.01 , respectively. The final model was reached using the remaining significant covariates.

\section{Model evaluation}

The final model was evaluated by goodness-of-fit plots, as follows: (1) observed values versus population- or individual-predicted values were plotted and (2) conditional weighted residuals [17] versus time after the first dose and population-predicted values were plotted.

Bootstrap validation $[18,19]$ was used to estimate the standard errors for the estimates and to evaluate the validity and robustness of the final model. Two hundred bootstrap replicates were generated by randomly nonparametric resampling the original dataset with replacement. The final model was fitted repeatedly to the 200
Table 1 Summary of subject data used in the modeling and simulation

\begin{tabular}{llll}
\hline & Zoledronic acid $(N=145)$ & Placebo $(N=161)$ & Total $(N=306)$ \\
\hline Sex & & & \\
$\quad$ Male & $11(7.6 \%)$ & $6(3.7 \%)$ & $17(5.6 \%)$ \\
$\quad$ Female & $134(92.4 \%)$ & $155(96.3 \%)$ & $289(94.4 \%)$ \\
Age (years) & $72.9 \pm 5.2$ & $72.9 \pm 5.3$ & $72.9 \pm 5.2$ \\
& {$[65-86]$} & {$[65-87]$} & {$[65-87]$} \\
Weight (kg) & $51.4 \pm 7.4$ & $53.1 \pm 8.4$ & $52.3 \pm 8.0$ \\
& {$[36.0-76.4]$} & {$[34.1-83.6]$} & {$[34.1-83.6]$} \\
Prior usage of bisphosphonate & & & \\
Never used & $132(91.0 \%)$ & $145(90.0 \%)$ & $277(90.5 \%)$ \\
Used with sufficient washout & $13(9.0 \%)$ & $16(10.0 \%)$ & $29(9.5 \%)$ \\
TRACP-5b (mU/dL) & $398.0 \pm 143.6$ & $403.9 \pm 152.0$ & $401.1 \pm 147.9$ \\
& {$[183-1240]$} & {$[157-1030]$} & {$[157-1240]$} \\
CTx (ng/mL) & $0.406 \pm 0.175$ & $0.404 \pm 0.190$ & $0.405 \pm 0.183$ \\
& {$[0.08-1.00]$} & {$[0.10-0.99]$} & {$[0.08-1.00]$} \\
u-NTx (nMBCE/mMCr.) & $54.52 \pm 21.43$ & $54.97 \pm 25.69$ & $54.76 \pm 23.73$ \\
Lumbar spine T-score (L2-L4) & {$[16.4-119.0]$} & {$[14.0-150.0]$} & {$[14.0-150.0]$} \\
& $-2.769 \pm 0.800$ & $-2.829 \pm 0.791$ & $-2.801 \pm 0.795$ \\
Lumbar spine BMD (L2-L4) $\left(\mathrm{g} / \mathrm{cm}^{2}\right)$ & {$[-5.51$ to -0.68$]$} & {$[-5.25$ to -0.25$]$} & {$[-5.51$ to -0.25$]$} \\
& $0.680 \pm 0.095$ & $0.674 \pm 0.094$ & $0.677 \pm 0.094$ \\
& {$[0.36-0.93]$} & {$[0.39-0.98]$} & {$[0.36-0.98]$} \\
\hline
\end{tabular}

Values are expressed as means \pm SD [range]

$T R A C P-5 b$ tartrate-resistant acid phosphatase $5 \mathrm{~b}, C T x$ C-telopeptide of type I collagen, $u$-NTx urinary Ntelopeptide of type I collagen, $B M D$ bone mineral density 
Fig. 2 Time profiles of bone resorption markers and the percentage change from baseline of the BMD following two annual doses of placebo (a) or ZOL (b). The expanded scale is shown in the inset plot. TRACP-5b tartrate-resistant acid phosphatase 5b, CTx C-telopeptide of type I collagen, $u$-NTx urinary Ntelopeptide of type I collagen, $B M D$ bone mineral density (a) Placebo
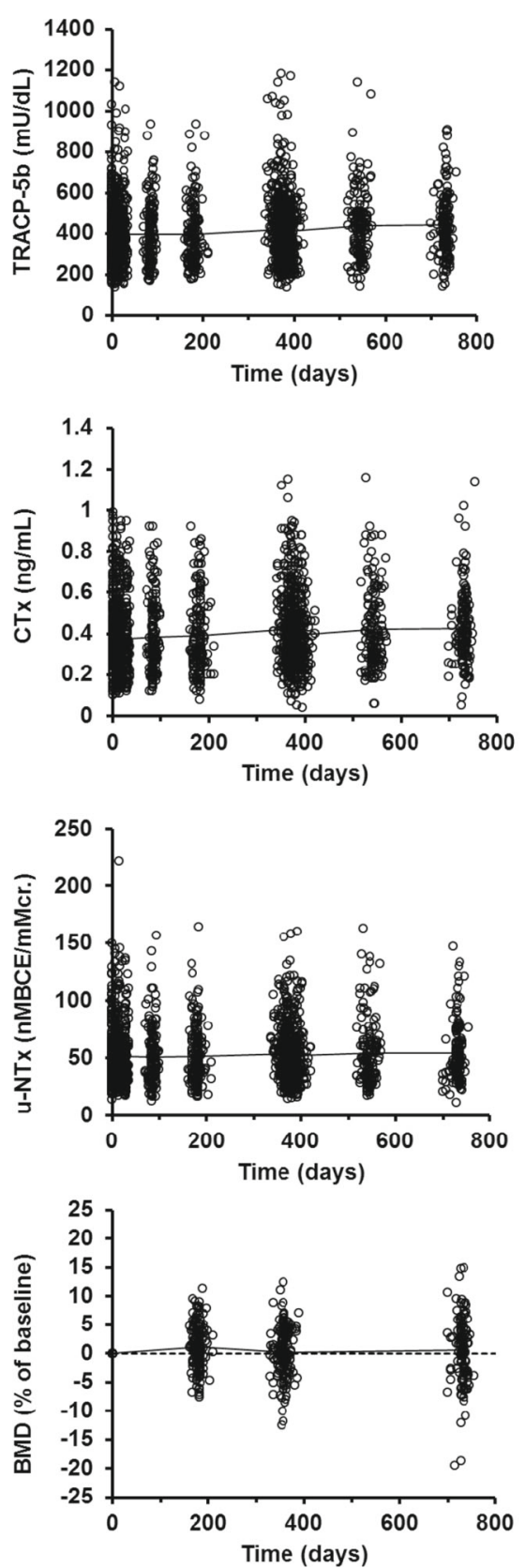

(b) ZOL
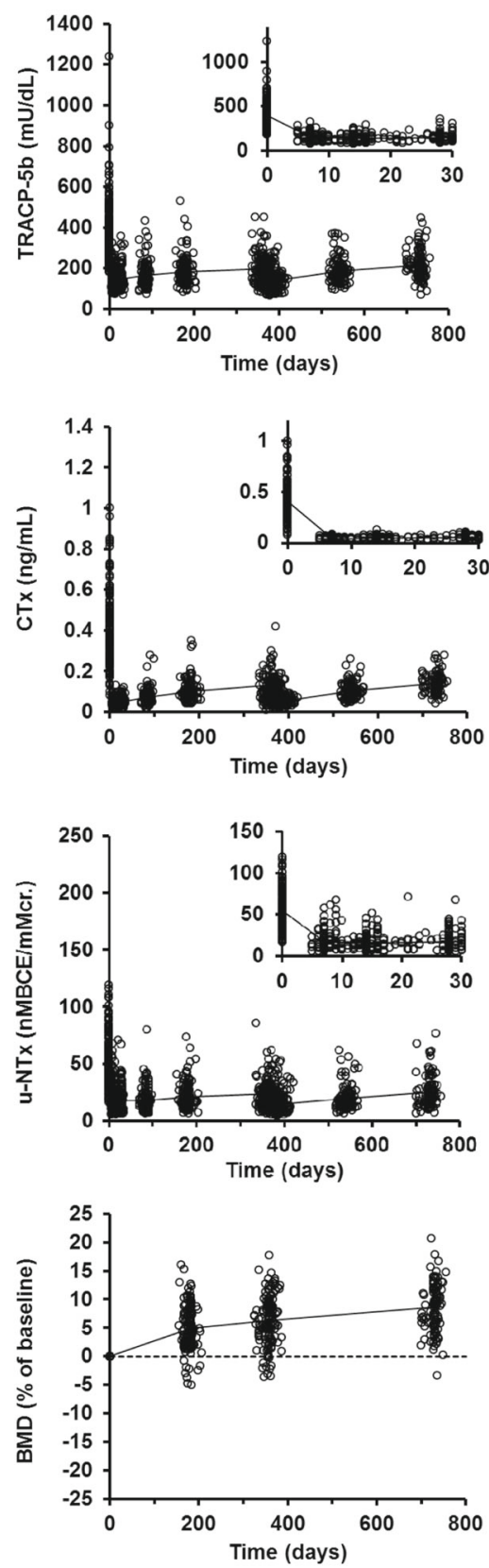

datasets. Successful estimation was defined as the normal completion of the Phoenix software. The means of parameter estimates calculated from the successful estimations were compared with the final parameter estimates obtained from the original dataset.

\section{Simulation based on the final model}

Two-year BMD was simulated following two annual doses of ZOL using baseline (bone resorption marker and $\mathrm{BMD}$ ) and 12-week (bone resorption marker) values as follows. To evaluate the prospective predictability of the model based on TRACP-5b, a visual predictive check [20] was performed. Ten thousand virtual patients were randomly simulated, and the time-dependent profiles of TRACP-5b and BMD were predicted using the final model. To compare predictability with other bone resorption markers, the same models were also developed with $\mathrm{CTx}$ or u-NTx instead of TRACP-5b, and empirical Bayes estimation was performed.

The relationships between the change from baseline in TRACP-5b at 12 weeks and the changes in T-scores and 
BMD at 2 years following two annual doses of ZOL were evaluated using simulation. Ten thousand virtual patients were randomly simulated, and the time-dependent profiles of TRACP-5b and BMD were predicted using the final model. A visual predictive check was performed to evaluate the predictability of the simulation. The percentages of patients whose BMD improved by more than $2.4 \%$ or a $\mathrm{T}$-score greater than -2.5 were estimated against three categories of TRACP-5b decreases $(100,200$, and $300 \mathrm{mU} / \mathrm{dL}$ ).

\section{Model development and simulation}

All modeling and simulation in this study were performed using a nonlinear mixed-effects modeling in Phoenix NLME 1.3 software (Pharsight Corporation, Certara, LP, Princeton, NJ) with the FOCE-ELS algorithm.

\section{Results}

\section{Patients and measured data}

Data from a total of 306 patients who had values for at least one bone resorption marker and BMD were used in this analysis, with 145 patients in the ZOL group and 161 in the placebo group. Patients' demographics are shown in Table 1. No significant differences between the ZOL and placebo groups were seen for any factors.

Bone resorption markers (TRACP-5b, CTx, and u-NTx, 3410 points), BMD (1146 points), and \%BMD were plotted against time since first administration (Fig. 2). In the ZOL group, bone resorption markers showed clear decreases from baseline even only a few weeks after the first administration. On the other hand, BMD and \%BMD showed improvements in the ZOL group, although there were no clear trends in the placebo group.

\section{Base model}

The model depicted in Fig. 1 gave an adequate prediction. Inter-individual random effects were estimated for $\mathrm{EKD}_{50}$, Emax, Slope, $\mathrm{T}_{50}, \mathrm{Ke} 0$, and Scale. Among the three bone resorption markers, TRACP-5b was selected as the best one to predict the BMD profile.

\section{Covariate exploration}

Statistically significant covariates were detected for the baseline TRACP-5b effect on $\mathrm{EKD}_{50}$, Slope, $\mathrm{T}_{50}$, and Scale. As far as Scale, baseline TRACP-5b was significant only in the ZOL
Table 2 Parameter values estimated by the mathematical model (TRACP-5b)

\begin{tabular}{|c|c|c|}
\hline Parameter (unit) & Estimate & $\mathrm{SE}^{\mathrm{a}}$ \\
\hline \multicolumn{3}{|l|}{ Bone resorption marker model } \\
\hline $\mathrm{KD}$ (1/day) & $3.719 \times 10^{-3}$ & $2.941 \times 10^{-5}$ \\
\hline Gamma & $4.583 \times 10^{-1}$ & $2.626 \times 10^{-3}$ \\
\hline $\mathrm{EKD}_{50}(\mathrm{mg} /$ day $)$ & $5.776 \times 10^{-3}$ & $3.800 \times 10^{-5}$ \\
\hline Kout (1/day) & $4.584 \times 10^{-1}$ & $3.592 \times 10^{-3}$ \\
\hline Slope (1/day) & $2.688 \times 10^{-4}$ & $2.877 \times 10^{-6}$ \\
\hline$E_{\max }$ & $-8.266 \times 10^{-2}$ & $2.053 \times 10^{-3}$ \\
\hline $\mathrm{T}_{50}($ day $)$ & $1.166 \times 10^{2}$ & 3.091 \\
\hline TRACP-5b baseline effect on $\mathrm{EKD}_{50}$ & -1.534 & $1.699 \times 10^{-2}$ \\
\hline TRACP-5b baseline effect on Slope & -1.350 & $2.093 \times 10^{-2}$ \\
\hline TRACP-5b baseline effect on $\mathrm{T}_{50}$ & -1.319 & $2.974 \times 10^{-2}$ \\
\hline$\sigma$ & $3.551 \times 10$ & $1.204 \times 10^{-1}$ \\
\hline \multicolumn{3}{|l|}{ Inter-individual variability } \\
\hline$\omega \mathrm{EKD}_{50}{ }^{2}$ & $2.255 \times 10^{-1}$ & $4.892 \times 10^{-3}$ \\
\hline$\omega_{\text {slope }}^{2}$ & $1.573 \times 10^{-1}$ & $3.136 \times 10^{-3}$ \\
\hline$\omega \mathrm{E}_{\max }^{2}$ & $8.584 \times 10^{-2}$ & $2.655 \times 10^{-3}$ \\
\hline$\omega \mathrm{T}_{50}^{2}$ & 2.095 & $3.662 \times 10^{-2}$ \\
\hline$\omega \mathrm{E}_{\max }$, slope & $-7.576 \times 10^{-2}$ & $2.806 \times 10^{-3}$ \\
\hline$\omega \mathrm{E}_{\max }, \mathrm{T}_{50}$ & $-8.732 \times 10^{-2}$ & $3.911 \times 10^{-3}$ \\
\hline wslope, $\mathrm{T}_{50}$ & $8.697 \times 10^{-2}$ & $4.525 \times 10^{-3}$ \\
\hline \multicolumn{3}{|l|}{ BMD model } \\
\hline $\operatorname{Ke} 0$ (1/day) & $3.802 \times 10^{-3}$ & $6.429 \times 10^{-4}$ \\
\hline Scale $\left(\left(\mathrm{g} / \mathrm{cm}^{2}\right) /(\mathrm{mU} / \mathrm{dL})\right)$ & $-2.521 \times 10^{-4}$ & $3.709 \times 10^{-5}$ \\
\hline TRACP-5b baseline effect on Scale & -1.112 & $2.635 \times 10^{-1}$ \\
\hline$\sigma$ & $2.447 \times 10^{-2}$ & $1.215 \times 10^{-3}$ \\
\hline \multicolumn{3}{|l|}{ Inter-individual variability } \\
\hline$\omega \mathrm{Ke} 0^{2}$ & $1.406 \times 10^{-3}$ & $2.964 \times 10^{-1}$ \\
\hline$\omega$ Scale $^{2}$ & $2.361 \times 10^{-8}$ & $1.028 \times 10^{-8}$ \\
\hline
\end{tabular}

${ }^{\mathrm{a}} \mathrm{SE}$ was obtained from Bootstrap validation

$S E$ standard error, $E K D_{50}$ the value of $\mathrm{KD} \times \mathrm{A}$ that led to $50 \%$ inhibition of bone resorption marker production rate constant, $E_{\max }$ the relative maximal effect, Gamma Hill coefficient, $K D$ first-order equilibrium rate constant, Kout bone resorption marker elimination rate constant, slope a linear time effect, $T_{50}$ the time at which the effect reached half of the maximal effect, $\mathrm{KeO}$ effect-compartment equilibrium rate constant, Scale ratio of change in bone resorption marker values to change in BMD

group. No covariates were statistically significant for Slope and $\mathrm{Ke} 0$.

\section{Goodness-of-fit}

Goodness-of-fit plots are shown in Supplemental Fig. 1. The plots of the observed vs. prediction values were almost evenly distributed centering on the 1:1 line. Most conditional weighted residuals were evenly distributed around 0 independently of the population-predicted values 


\section{(a) Placebo}

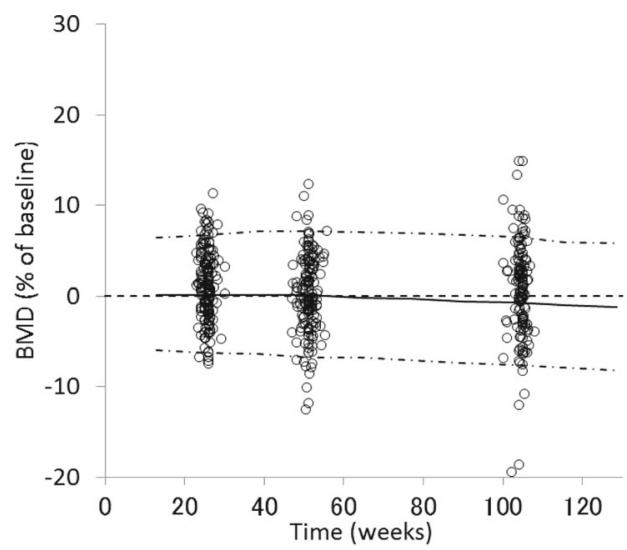

Fig. 3 Predicted percentage change from baseline of the BMD (\%BMD) simulated from the data at baseline and 12 weeks. Ten thousand virtual BMDs following two annual doses of ZOL were simulated from background data (baseline of TRACP-5b and BMD) and early values

and time after the first dose. The final parameter estimates are shown in Table 2.

\section{Simulation}

Using the final model, BMD up to 2 years was predicted using only the background values (baseline of TRACP-5b and BMD) and the early values (TRACP-5b at 12 weeks). $\% \mathrm{BMD}$ was calculated using predicted BMD. A visual predictive check showed that the simulated $90 \%$ prediction interval almost covered the observed \%BMD distribution, though it was simulated with only three values (Fig. 3). To compare predictability with other bone resorption markers, the same models with CTx or u-NTx (Supplemental Table 1) instead of TRACP-5b were also developed, and empirical Bayes estimation was performed using the baseline and 12-week values of each bone resorption marker. TRACP-5b model showed the best predictive performance among the three kinds of bone

Table 3 The relationship between the change in TRACP-5b from baseline to 12 weeks and the predicted percentage T-score or BMD improvement at 2 years

\begin{tabular}{llll}
\hline Predicted value at 2 years & \multicolumn{2}{l}{$\begin{array}{l}\text { Change in TRACP-5b from baseline to } \\
12 \text { weeks }\end{array}$} \\
\cline { 2 - 4 } & $100 \mathrm{mU} / \mathrm{dL}$ & $200 \mathrm{mU} / \mathrm{dL}$ & $300 \mathrm{mU} / \mathrm{dL}$ \\
\hline T-score $>-2.5$ & $19.6 \%$ & $26.6 \%$ & $34.2 \%$ \\
$\% \mathrm{BMD}>2.4 \%$ & $68.5 \%$ & $76.9 \%$ & $82.7 \%$ \\
\hline
\end{tabular}

BMD of 10,000 patients at 2 years following two annual doses were randomly simulated using the baseline BMD and TRACP-5b values of patients with baseline T-scores less than -2.5 . T-scores and \%BMD were calculated from the predicted BMD

$\% B M D$ percentage change from baseline of the BMD (b) ZOL

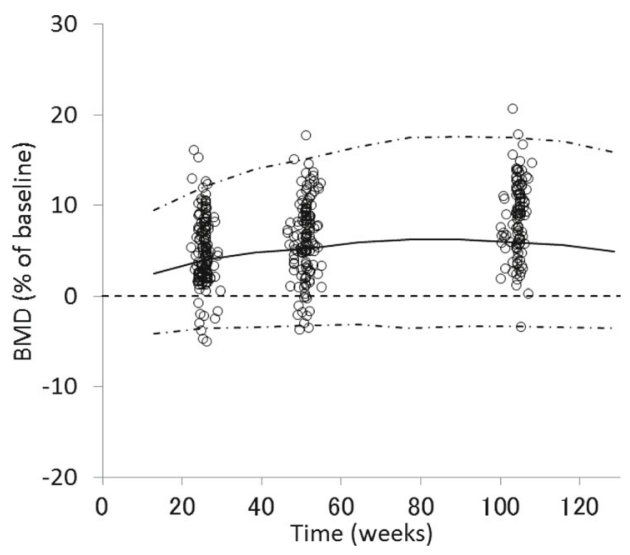

(TRACP-5b values at 12 weeks). \%BMD was calculated from simulated BMD. The solid lines indicate the medians of simulated $\%$ BMD. The upper and lower chain lines indicate the 95th and 5th percentiles of simulated \%BMD, respectively

resorption markers statistically, whereas they showed almost the same predictability by visual inspection (Supplemental Fig. 2).

The relationships between the change from baseline in TRACP-5b at 12 weeks and the changes in T-scores and BMD at 2 years following two annual doses of ZOL were examined. A visual predictive check was performed to evaluate the predictability of the simulation. Virtual \%BMD to 2 years following two annual doses of 10,000 patients with baseline T-scores less than -2.5 were simulated and plotted against the TRACP-5b change from baseline to 12 weeks. The simulated $90 \%$ prediction interval showed good coverage for the observed \%BMD distribution with some points falling outside the interval (Supplemental Fig. 3). The percentages of the patients whose T-score greater than -2.5 or BMD improved by more than $2.4 \%$ against three categories of TRACP-5b decrease $(100,200$, and $300 \mathrm{mU} / \mathrm{dL})$ were estimated by this simulation (Table 3 ).

\section{Discussion}

In this analysis, mathematical models were constructed using TRACP-5b serum concentrations and lumbar spine BMD data from 306 patients with primary osteoporosis treated with ZOL to predict BMD at up to 2 years. The constructed models showed that good predictability, in particular, BMD up to 2 years could be predicted from background data (baseline TRACP-5b and BMD) and early values (TRACP-5b values at 12 weeks) (Fig. 3 and Supplemental Fig. 2).

For the BMD prediction, Hasegawa et al. constructed an exposure-response model after administration of ONO-5334, a selective inhibitor of cathepsin K [12]. In addition, Nakai et al. developed a population pharmacodynamic model to 
relate urinary $\mathrm{CTx}$ to $\mathrm{BMD}$ response after treatment with ibandronate, adequately predicting \%BMD at 3 years after treatment [11]. The present model showed almost the same predictability for BMD. However, these previous efforts did not focus on the use of bone turnover markers at the initial stage of drug treatment to predict the BMD response years later. In the present analysis, BMD at up to 2 years could be predicted using only background (baseline TRACP-5b and BMD) and early (TRACP-5b at 12 weeks) values, which supports the prediction of long-term BMD profiles using only very early stage data after first dosing. Diez-Perez et al. suggested that PINP and CTx after 3 months of therapy could be used to screen for the treatment effect of oral bisphosphonates [8]. These findings, in which the changes in bone turnover markers in the early phase of bisphosphonate treatment predict future changes in BMD, have also been observed in cases receiving a bone formation agent. Namely, Chen et al. reported that early changes in bone turnover markers, PICP at 1 month and PINP at 3 months, correlated with the 18month BMD response to daily teriparatide therapy in women with postmenopausal osteoporosis [7]. In addition to this study, Tsujimoto et al. proposed an algorithm using PINP to monitor patients, since there was a strong relationship between early changes in PINP and later changes in lumbar spine \%BMD during teriparatide therapy [9]. These three reports suggested that the long-term BMD profiles might be predictable using values of bone turnover markers in the early stage, regardless of the mode of therapy, and they thus support the present findings.

In the present analysis, three base models were constructed using each of the bone resorption markers, TRACP-5b, CTx, and $\mathrm{u}-\mathrm{NTx}$. Though the simulation of 2-year BMD from early values of CTx or u-NTx gave almost the same predictability as that of TRACP-5b by visual inspection (Supplemental Fig. 2), the TRACP-5b model was superior based on statistical significance. The statistical superiority of TRACP-5b could be due to the possibility that the biological variability of TRACP-5b is smaller than that of CTx, as has been previously reported [21]. Our established models also supported this possibility as the intra-individual variability of the TRACP-5b model was smaller than that of the CTx model (8.9 vs. $13.3 \%$; Supplemental Table 2). It is therefore suggested that the TRACP-5b model is the best indicator to describe the BMD profile after treatment with $\mathrm{ZOL}$.

In addition, TRACP-5b has been shown to be a good marker for serum bone resorption, since it is not affected by renal dysfunction [22]. Furthermore, its intra-individual variability is low because it is not affected by food intake [21]. Therefore, the present model may be useful to predict BMD in patients with normal renal function or in patients with renal dysfunction, which should be confirmed in a further study.

The relationship between the change from baseline in TRACP-5b at 12 weeks and the changes in T-scores and
$\% \mathrm{BMD}$ at 2 years following two annual doses of ZOL were estimated. The percentages of patients whose BMD improved by more than $2.4 \%$ as a short-term treatment goal $[6,23]$ or achieved T-score greater than -2.5 as a long-term treatment goal $[4,5]$ against three categories of TRACP-5b decrease $(100,200$, and $300 \mathrm{mU} / \mathrm{dL})$ have been shown to be predictable by this simulation (Table 3 ).

In the present model, there are two limitations. Firstly, the present study population was patients who met the selection criteria and were given supplemental calcium and vitamin D. Thus, the generalizability of the present results must be clarified in the real world. Another is that the model could not be applied to patients who were switched from other bisphosphonates, because the model was developed from the ZONE study in which all patients never used bisphosphonates or used bisphosphonates with sufficient washout. This should also be tested in a further study.

In this study, the focus was on future values of BMD as the expected benefit of ZOL treatment, because BMD is generally accepted as the standard measure of fracture risk, which is the clinical outcome of osteoporosis [2]; however, it still does not entirely assess fracture risk [1]. Bell et al. demonstrated that early changes of hip BMD and serum NTx after ZOL treatment were ranked highly for prediction of clinical fractures [24]. It was thought that further study was needed to predict fracture risk quantitatively from the early response to $\mathrm{ZOL}$ treatment.

In conclusion, this is the first model to predict BMD at 2 years following two annual doses of ZOL using patients' baseline data and the early response of TRACP-5b. This model allows us to inform patients of their predicted response at the initial stage of ZOL treatment.

Acknowledgments The authors would like to thank Rika Oishi and Satoshi Tanaka for their fruitful comments and suggestions and Risa Tanaka and Yuri Ota for their technical support.

\section{Compliance with ethical standards}

This study was conducted in compliance with the World Medical Association Declaration of Helsinki-Ethical Principles for Medical Research Involving Human Subjects and Good Clinical Practice. The protocol was approved by the institutional review board at each study site. Written, informed consent was obtained from all subjects before enrollment in the study.

Conflicts of interest Yoko Mori, Atsushi Ose, Masashi Serada, and Masashi Ishiguro are employees of Asahi Kasei Pharma. Hidefumi Kasai and Masataka Shiraki received consulting fees from Asahi Kasei Pharma. Yusuke Tanigawara received research funds from Asahi Kasei Pharma.

Ethical approval All procedures performed in studies involving human participants were in accordance with the ethical standards of the institutional and/or national research committee and with the 1964 Helsinki declaration and its later amendments or comparable ethical standards.

Informed consent Informed consent was obtained from all individual participants included in the study. 
Open Access This article is distributed under the terms of the Creative Commons Attribution-NonCommercial 4.0 International License (http:// creativecommons.org/licenses/by-nc/4.0/), which permits any noncommercial use, distribution, and reproduction in any medium, provided you give appropriate credit to the original author(s) and the source, provide a link to the Creative Commons license, and indicate if changes were made.

\section{References}

1. NIH Consensus Development Panel on Osteoporosis Prevention D, Therapy (2001) Osteoporosis prevention, diagnosis, and therapy. JAMA 285(6):785-795. https://doi.org/10.1001/jama.285.6.785

2. Unnanuntana A, Gladnick BP, Donnelly E, Lane JM (2010) The assessment of fracture risk. J Bone Joint Surg Am 92(3):743-753. https://doi.org/10.2106/JBJS.I.00919

3. Kanis J (2007) Assessment of osteoporosis at the primary healthcare level. University of Sheffield, WHO Collaborating Centre for Metabolic Bone Diseases

4. Kuroda T, Shiraki M, Shiraki Y, Tanaka S (2012) The importance of absolute bone mineral density in the assessment of antiresorptive agents used for the prevention of osteoporotic fractures. J Clin Densitom 15(4):392-398. https://doi.org/10.1016/j.jocd.2012.02. 005

5. Cummings SR, Cosman F, Lewiecki EM et al (2017) Goal-directed treatment for osteoporosis: a progress report from the ASBMRNOF working group on goal-directed treatment for osteoporosis. J Bone Miner Res 32:3-10

6. Diez-Perez A, Adachi JD, Agnusdei D et al (2012) Treatment failure in osteoporosis. Osteoporos Int 23(12):2769-2774. https://doi. org/10.1007/s00198-012-2093-8

7. Chen P, Satterwhite JH, Licata AA, Lewiecki EM, Sipos AA, Misurski DM, Wagman RB (2005) Early changes in biochemical markers of bone formation predict BMD response to teriparatide in postmenopausal women with osteoporosis. J Bone Miner Res 20(6):962-970. https://doi.org/10.1359/JBMR.050105

8. Diez-Perez A, Naylor KE, Abrahamsen B et al (2017) International osteoporosis foundation and European calcified tissue society working group. Recommendations for the screening of adherence to oral bisphosphonates. Osteoporos Int 28(3):767-774. https://doi. org/10.1007/s00198-017-3906-6

9. Tsujimoto M, Chen P, Miyauchi A, Sowa H, Krege JH (2011) PINP as an aid for monitoring patients treated with teriparatide. Bone 48: 798-803

10. Nakamura T, Fukunaga M, Nakano T et al (2017) Efficacy and safety of once-yearly zoledronic acid in Japanese patients with primary osteoporosis: two-year results from a randomized placebocontrolled double-blind study (ZOledroNate treatment in efficacy to osteoporosis; ZONE study). Osteoporos Int 28:389-398

11. Nakai K, Iida S, Tobinai M, Hashimoto J, Kawanishi T (2015) Application of modeling and simulation to a long-term clinical trial: a direct comparison of simulated data and data actually observed in
Japanese osteoporosis patients following 3-year ibandronate treatment. Clin Pharmacokinet 54:295-304

12. Hasegawa C, Kastrissios H, Monteleone J et al (2014) Modeling and simulation of bone mineral density response from a phase 2 study of ONO-5334, a new cathepsin K inhibitor, to support dose selection in osteoporosis. J Clin Pharmacol 54:937-948

13. Peterson MC, Riggs MM (2012) Predicting nonlinear changes in bone mineral density over time using a multiscale systems pharmacology model. CPT Pharmacometrics Syst Pharmacol 1(11):e14. https://doi.org/10.1038/psp.2012.15

14. Marathe DD, Marathe A, Mager DE (2011) Integrated model for denosumab and ibandronate pharmacodynamics in postmenopausal women. Biopharm Drug Dispos 32:471-481

15. Peterson MC, Riggs MM (2010) A physiologically based mathematical model of integrated calcium homeostasis and bone remodeling. Bone 46(1):49-63. https://doi.org/10.1016/j.bone.2009.08. 053

16. Marathe A, Peterson MC, Mager DE (2008) Integrated cellular bone homeostasis model for denosumab pharmacodynamics in multiple myeloma patients. J Pharmacol Exp Ther 326(2):555562. https://doi.org/10.1124/jpet.108.137703

17. Hooker AC, Staatz CE, Karlsson MO (2007) Conditional weighted residuals (CWRES): a model diagnostic for the FOCE method. Pharm Res 24(12):2187-2197. https://doi.org/10.1007/s11095007-9361-x

18. Parke J, Holford NH, Charles BG (1999) A procedure for generating bootstrap samples for the validation of nonlinear mixed-effects population models. Comput Methods Prog Biomed 59(1):19-29. https://doi.org/10.1016/S0169-2607(98)00098-4

19. Efron B, Tibshirani RJ (1993) An introduction to the bootstrap (monographs on statistics and applied probability). Chapman \& Hall (new York)

20. Holford NH (2005) The visual predictive check: superiority to standard diagnostic (Rorschach) plots (http://www.page-meeting.org/? abstract=738). PAGE abstracts of the annual meeting of the population approach Group in Europe 14:

21. Hannon RA, Clowes JA, Eagleton AC, Al Hadari A, Eastell R, Blumsohn A (2004) Clinical performance of immunoreactive tartrate-resistant acid phosphatase isoform $5 \mathrm{~b}$ as a marker of bone resorption. Bone 34(1):187-194. https://doi.org/10.1016/j.bone. 2003.04.002

22. Yamada S, Inaba M, Kurajoh M, Shidara K, Imanishi Y, Ishimura E, Nishizawa Y (2008) Utility of serum tartrate-resistant acid phosphatase (TRACP5b) as a bone resorption marker in patients with chronic kidney disease: independence from renal dysfunction. Clin Endocrinol 69:189-196

23. Shiraki M, Ueda S, Sugimoto T, Kuroda T, Nakamura T (2016) Treatment responses with once-weekly teriparatide therapy for osteoporosis. Osteoporos Int 27:3057-3062

24. Bell KJ, Hayen A, Glasziou P, Irwig L, Eastell R, Harrison SL, Black DM, Bauer DC (2016) Potential usefulness of BMD and bone turnover monitoring of Zoledronic acid therapy among women with osteoporosis: secondary analysis of randomized controlled trial data. J Bone Miner Res 31(9):1767-1773. https://doi.org/10. 1002/jbmr.2847 